\title{
What new cell biology findings could bring to therapeutics: is it time for a phenome-project in Toxoplasma gondii?
}

\author{
Markus Meissner/ ${ }^{+}$, Katrin Klaus \\ Hygieneinstitut, Departament of Parasitology, University Hospital Heidelberg, ImNeuenheimer Feld 324, D-69120 Heidelberg, Germany
}

"If you know the enemy and know yourself, you need not fear the result of a hundred battles. If you know yourself but not the enemy, for every victory gained you will also suffer a defeat" (SunTzu the Art of War, 544-496 BC). Although written for the managing of conflicts and winning clear victories, this basic guideline can be directly transferred to our battle against apicomplexan parasites and how to focus future basic research in order to transfer the gained knowledge to a therapeutic intervention stratey. Over the last two decades the establishment of several key-technologies, by different groups working on Toxoplasma gondii, made this important human pathogen accessible to modern approaches in molecular cell biology. In fact more and more researchers get attracted to this easy accessible model organism to study specific biological questions, unique to apicomplexans. This fascinating, unique biology might provide us with new therapeutic options in our battle against apicomplexan parasites by finding its Achilles' heel. In this article we argue that in the absence of a powerful high throughput technology for the characterisation of essential gene of interests a coordinated effort should be undertaken to convert our knowledge of the genome into one of the phenome.

Key words: Toxoplasma - gene regulation - conditional mutagenesis - Apicomplexa

If one mention "apicomplexan parasites" most people in the world think of horrifying diseases like malaria or toxoplasmosis. Although a central aim of research should be the rapid development of new drugs and vaccines against these pathogens using "traditional" screening methods, many researchers study these "bugs" for the sake of science itself. In fact, over the last two decades, boosted by the establishment of several key technologies (Meissner et al. 2007b), more and more researchers discover apicomplexans as attractive model organisms to study specific questions, like plastid biology, secretory pathway, cell motility or host pathogen interactions (Gubbels \& Striepen 2004, Kim \& Weiss 2004, Frischknecht et al. 2006, Vaishnava \& Striepen 2006, Carruthers \& Boothroyd 2007, Striepen et al. 2007).

However, the main challenge is to determine on what research should be focused in the future. Which questions should be raised and studied specifically in apicomplexan parasites, because they are of interest, both for fundamental biology and development of future therapies. In consequence, we also need to decide which questions can be neglected or postponed, since they have likely been answered previously in more accessible

Financial support: $\mathrm{MM}$ is funded by the BioFuture-Programm (0311897) of the German Ministry of Science and Education. KK is funded by the SFB544 of the German Research Foundation.

+ Corresponding author: markus.meissner@med.uni-heidelberg.de Received 10 October 2008

Accepted 3 December 2008 model organisms, such as yeast, Drosophila, Homo. Alternatively, should we ignore previous findings in other organisms and study every gene identified in the genome to characterise the phenome of this parasite. This approach would include highly conserved and unique genes in the analysis.

In the later case we need to establish more powerful high throughput methodologies that enable us to look at every single gene in an easy and effective way. However, in the absence of functional siRNA-methods (Meissner et al. 2007b), it is hard to imagine how such an approach is feasible. One alternative would be the establishment of a collaborative research network, with the aim to characterise every gene of interest (GOI) in a systematic approach, using the technologies currently available and sharing the load of work involved. While this approach is very work intense and requires considerable resources it would be a good investment into the future to generate a library of mutant parasites that is accessible to the community and represents a resource for the further study of specific questions. At this point it is worth to point out (once again) that in fact these approaches were very successful in other model organisms, such as yeast [Prophecy: (Fernandez-Ricaud et al. 2007), mouse (Fernandez-Ricaud et al. 2007) or plants (Edwards \& Batley 2004)].

In this article we would like to briefly review the current tools available to genetically manipulate the parasite to characterise GOIs and discuss if a combination of these tools would facilitate a "phenome" approach in Toxoplasma gondii and what technologies could be exploited in the future for this effort. We would like to discuss how the field could make the next step in molecular biology in order to convert our knowledge of the genome to one of the phenome by establishing a collaborative network. 


\section{Molecular tools for identification and characterisation of essential genes: a slow "one-by-one" approach}

After the first successful transfection of $T$. gondii 15 years ago (Soldati \& Boothroyd 1993), several groups continuously established reverse genetic tools that allow stable transfection, homologous and non-homologous (random) recombination, fluorescent labelling of parasites ectopic regulation of essential genes or complementation cloning (Meissner et al. 2007a). During the asexual life cycle, $T$. gondii is haploid, a fact that makes the removal of a gene via homologous recombination straight forward. However, in case the GOI is essential during the asexual life cycle, the generation of mutant parasites is impossible. In order to characterise essential factors in detail, three strategies are currently being employed with great success: (i) generation of temperature sensitive mutants followed by complementation cloning to identify the mutated gene (Gubbels et al. 2008); (ii) employment of a tetracycline inducible (Tet-inducible) transactivator system (Meissner et al. 2002) and (iii) a recently established degradation system (ddFKBP-system) that allows the rapid regulation of a protein of interest (Herm-Gotz et al. 2007). However, as mentioned above, the absence of a "real" high throughput system, such as siRNA based methods (Ullu et al. 2004), is seriously hampering a genome wide approach as performed in other eukaryotes.

The three systems currently employed have obvious advantages and disadvantages that need to be taken into consideration before a GOI is attempted to be analysed. Although the generation of temperature sensitive mutants appears to be relatively straight forward, the isolation of the respective locus might be complicated in case several genes have been mutated or the respective mutation has a dominant effect. However, with the optimisation of complementation technologies (Striepen et al. 2002, Gubbels et al. 2008), this technique has been currently demonstrated to be very powerful for the identification and isolation of novel essential genes (Gubbels et al. 2008). The obvious advantage of this strategy is that hypothetical genes that might otherwise be neglected in targeted approaches will be identified with equal probability. The Tet-inducible transactivator system is currently the most robust method to generate conditional mutants for essential genes in a targeted approach and appears to be especially well suited for the characterisation of factors involved in invasion and apicoplast biology (Meissner et al. 2002, Mital et al. 2005, Huynh \& Carruthers 2006, Mazumdar et al. 2006, Kessler et al. 2008, Plattner et al. 2008) whereas employment of the system for other essential GOIs might result only in weak phenotypes (Fleige et al. 2008) or might not be possible at all (unpublished observations). A major advantage of the Tet-inducible system is that virtually any GOI can be regulated whereas regulation at the protein level using ddFKBP might not work for proteins targeted to the secretory system (Herm-Gotz et al. 2007, and unpublished observations). However, apart from certain inefficiency problems, the application of the Tet-inducible system is very work intense. Three independent stable transfection and selections for clonal parasite populations are required, which takes at least three months for a single gene and hence is not applicable in a genome wide approach.

In contrast, the ddFKBP-system is especially well suited for the characterisation of essential proteins in a dominant negative approach. Here a single stable transfection is sufficient to generate the respective parasite mutant. Other advantages of this system are the rapid induction kinetics and the possibility to tune the level of protein level simply via adding different amounts of inducer (Herm Gotz et al. 2007).

The generation of parasite mutants using random insertion mutagenesis is another promising approach. However, in this approach only non-essential genes for tachyzoite growth in vitro can be identified. Hence this strategy has been mainly employed to identify genes required for differentiation from tachyzoites to bradyzoites (Matrajt et al. 2002, Vanchinathan et al. 2005). In the future, a combination of ectopic gene regulation systems with random insertional mutagenesis might be possible. In fact, we recently generated a parasite strain expressing a regulable Flp-recombinase that should allow temporal control of site-specific recombination. Our hope is that saturation of the genome with Frt-sites via random insertion and subsequent activation of Flp will result in an easy to analyse mutant population of parasites (unpublished observations).

In conclusion, several strategies have been developed by several groups that allow characterisation of essential genes. However, so far researchers in the field aim to target a specific set of candidate genes. With more and more established easy-to-use tools the chance of redundant work on one and the same GOI increases. Therefore the next step should be taken: establishment of a phenome-network that aims to generate parasite mutants for GOI at large scale in order to provide a valuable resource for the community.

\section{A rapid "hand in hand" approach to tackle the phe- nome}

As summarised above, the generation and phenotypisation of mutants impaired in essential steps during the asexual life cycle combined with a complementation strategy is a very promising approach and has been successfully applied for a forward genetic screen to identify novel essential genes involved in cell cycle regulation (Gubbels et al. 2008). Similar strategies for other mutants (i.e., host cell invasion) would perfectly complement a targeted approach for promising GOIs. In addition, the further optimisation and development of reverse genetic tools could lead to more time efficient screening protocols.

For the characterisation of GOI in a targeted approach a systematic, coordinated analysis of the existing databases needs to be performed to generate a priority list that includes the most promising factors. For example, a focus on the "drugable" genome could be envisioned or the systematic analysis of certain unique organelles/ pathways/mechanisms like invasion, vesicle trafficking, apicoplast biology or host-parasite interactions. Sub- 
sequently, conditional gene expression systems can be employed to generate and initially analyse mutants for the respective GOI in a medium throughput. Given the huge amount of work, this effort needs to be efficiently coordinated by several research groups.

The ddFKBP-system would provide a good starting point for such a systematic approach with obvious limitations, since it is a one component system, meaning that a single transfection would be sufficient to establish a mutant with a regulable, dominant-negative/active copy of the GOI. Therefore, the establishment of an efficient medium-throughput approach for the characterisation of certain GOIs should be feasible. In fact, we recently generated mutants for different classes of proteins as a proof of concept, like GTPases, protein-kinase or motor proteins (myosins, dyneins, kinesins).

Obviously, this approach can not be applied for all GOIs, since minimum information about the GOI needs to be available beforehand to predict mutations that lead to a trans-dominant version of the GOI. However, in combination with proper bioinformatic analysis, an extensive list of GOIs could be assembled that contains several promising drug and vaccine candidates that await their future validation.

Currently, the ddFKBP-system allows efficient regulation of cytosolic proteins, whereas secretory proteins (especially soluble ones) can not be tackled with this system (our unpublished observations). Therefore, we are currently attempting to combine the ddFKBP-system with a site-specific recombination system to remove a GOI after addition of inducer and hence add temporal control to the system. Until this novel tool can be efficiently applied, the Tet-inducible transactivator system can be employed to "close the gap", since it has been demonstrated to work reliable for several proteins destined for the secretory pathway.

The next step after generation of a conditional mutant should be the detailed characterisation of the respective phenotype. Given the limited output of conditional mutants and the work-intensity involved for its establishment in the past, the usual approach was to establish and analyse a given mutant irrespective of the resulting phenotype. Now that the establishment of mutants can be achieved in a more time efficient manner, it gives researchers the freedom to concentrate on mutants with expected, surprising and interesting phenotypes, whereas others can be stored for later characterisation or made available to the community. Therefore, a central phenome database, possibly integrated into ToxoDB (www. toxoDB.org) should be established where information about novel mutants can be submitted and made available to the field. Similar databases exist for other organisms and could be easily adapted (http://prophecy.lundberg. gu.se/). The figure shows schematically how such an integrated approach could be organised. Briefly mutants are generated in random screens (temperature sensitive mutants or random insertional mutagenesis) and the respective mutation is identified. In parallel, coordinated bioinformatic database mining generates a list of priority candidates that are systematically characterised using

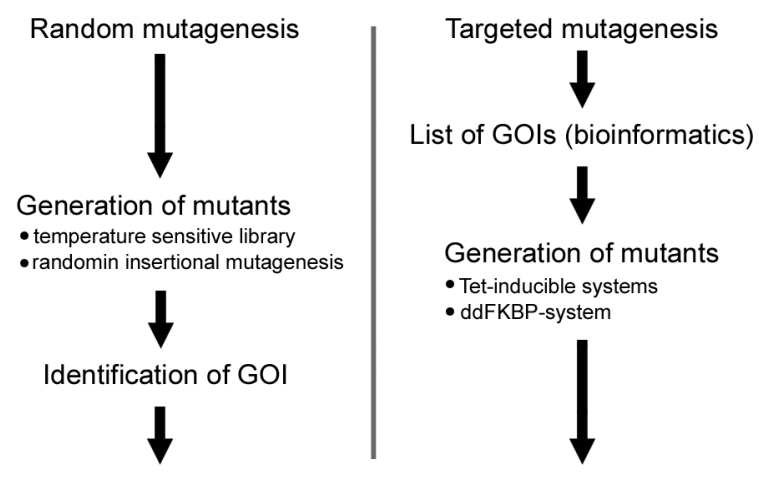

Phenotypisation and submission to Open Access Database

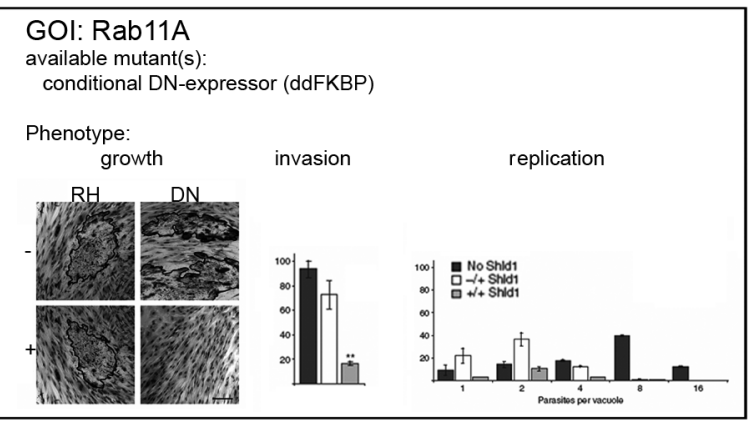

Schematic for a possible coordinated approach to characterize the phenome of Toxoplasma gondii. The phenotypic characterization of the small GTPase Rab11A is shown as an example for how mutants could be presented in an open access database. Modified from HermGotz et al. 2007.

conditional gene expression systems (ddFKBP or Tetsystem). Mutants then need to be characterised in a standardised and rapid assays. In our opinion, the minimal information that needs to be provided is if the respective mutant shows a general growth defect and if this is due to the intracellular development of the parasite or due to a defect in host cell invasion. Finally, a summary of this information can be entered in an open access database and made available to the community (Figure).

\section{An "easy" start: systematic analysis of factors in- volved in vesicular trafficking}

As mentioned above, the ddFKBP-system is especially well suited to characterise essential proteins in a dominant negative approach. For this, a single transfection is sufficient in order to generate a mutant parasite strain. However, minimal information about the protein is required in order to generate appropriate expression constructs that lead to a trans-dominant phenotype. We are currently focusing on proteins containing GTPase domains and other P-loop containing proteins (i.e. dynamins, motor proteins). The advantage here is that the domain architecture of these proteins allows the easy introduction of a single point mutation to generate a transdominant version of the respective protein. In fact, we generated a whole set of mutants for small Rab-GTPases that can be efficiently regulated using ddFKBP-system (Herm-Gotz et al. 2007) and in preparation. 
Rab proteins represent the largest family of monomeric ras-related GTPases and play a crucial role in membrane transport to and from organelles (Grosshans et al. 2006). Interestingly, while in higher eukaryotes more then 60 members of this family can be identified, apicomplexan parasites possess a reduced set of Rab-proteins that must be sufficient to organise specific vesicular trafficking in these parasites (Langsley et al. 2008, Sheiner \& Soldati-Favre 2008). Whereas Toxoplasma encodes 14 different Rabs, Plasmodia have 11 and Theileria just nine putative genes for Rab proteins. Currently, the role of two Rab-proteins (Rab5A and Rab6) has been analysed in more detail in T.gondii, by expressing either dominant negative or dominant active versions of the respective Rab (Robibaro et al. 2002, Stedman et al. 2003). However, the limitation of expressing trans-dominant versions of a protein in transient transfections is that the resulting phenotype might be non-specific and is in general hard to study in detail. Employment of the ddFKBP-system now gives us the chance to generate a clonal parasite line expressing each Rab-proteins in a regulated and tuneable manner, which results in a population of parasites that behaves identical, as shown for Rab1 and Rab11A (Herm-Gotz et al. 2007). Given the reduced core set of proteins involved in vesicular trafficking, we are optimistic that this approach will allow a systematic dissection of protein trafficking, organellar biogenesis and endocytosis in a relatively fast and straight forward approach. In this respect, it will be especially interesting to elucidate mechanisms involved in the synthesis of specialised secretory organelles and structures that evolved specifically in apicomplexan parasites in order to adapt to a parasitic lifestyle a question that is of high interest in the light of more general biology but also in order to identify and validate drug targets for the future therapeutic intervention.

Over the past decades, several key-technologies have been developed that allow the generation of parasite mutants for GOIs. However, all of these tools have one major disadvantage: they are work intense and do not allow a generation of mutants in a high throughput approach. However, a coordinated combination of the available technologies would allow at least the systematic characterisation of a subset of GOIs. Therefore the time has come to coordinate the efforts of different groups to reach the next logical goal after the characterisation of the genome, transcriptome and proteome: the phenome of $T$. gondii has come within our reach and will cast many new challenges for the next 100 years of research on T. gondii.

\section{ACKNOWLEDGEMENT}

To Dr. Friedrich Frischknech and Carolina Agop-Nersesian, for helpful comments on the manuscript.

\section{REFERENCES}

Carruthers V, Boothroyd JC 2007. Pulling together: an integrated model of Toxoplasma cell invasion. Curr Opin Microbiol 10: 83-89.

Edwards D, Batley J 2004. Plant bioinformatics: from genome to phenome. Trends Biotechnol 22: 232-237.
Fernandez-Ricaud L, Warringer J, Ericson E, Glaab K, Davidsson P, Nilsson F, Kemp GJ, Nerman O, Blomberg A 2007. PROPHECY-a yeast phenome database, update 2006. Nucleic Acids Res 35: D463-467.

Fleige T, Pfaff N, Gross U, Bohne W 2008. Localisation of gluconeogenesis and tricarboxylic acid (TCA)-cycle enzymes and first functional analysis of the TCA cycle in Toxoplasma gondii. Int $J$ Parasitol 38: 1121-1132.

Frischknecht F, Renaud O, Shorte SL 2006. Imaging today's infectious animalcules. Curr Opin Microbiol 9: 297-306.

Grosshans BL, Ortiz D, Novick P 2006. Rabs and their effectors: achieving specificity in membrane traffic. Proc Natl Acad Sci USA 103: 11821-11827.

Gubbels MJ, Lehmann M, Muthalagi M, Jerome ME, Brooks CF, Szatanek T, Flynn J, Parrot B, Radke J, Striepen B, White MW 2008. Forward genetic analysis of the apicomplexan cell division cycle in Toxoplasma gondii. PLoS Pathog 4: e36.

Gubbels MJ, Striepen B 2004. Studying the cell biology of apicomplexan parasites using fluorescent proteins. Microsc Microanal 10: 568-579.

Herm-Gotz A, Agop-Nersesian C, Munter S, Grimley JS, Wandless TJ, Frischknecht F, Meissner M 2007. Rapid control of protein level in the apicomplexan Toxoplasma gondii. Nat Methods 4: 1003-1005.

Huynh MH, Carruthers VB 2006. Toxoplasma MIC2 is a major determinant of invasion and virulence. PLoS Pathog 2: 753-762.

Kessler H, Herm-Gotz A, Hegge S, Rauch M, Soldati-Favre D, Frischknecht F, Meissner M 2008. Microneme protein 8-a new essential invasion factor in Toxoplasma gondii. J Cell Sci 121: 947-956.

Kim K, Weiss LM 2004. Toxoplasma gondii: the model apicomplexan. Int J Parasitol 34: 423-432.

Langsley G, van Noort V, Carret C, Meissner M, de Villiers EP, Bishop R, Pain A 2008. Comparative genomics of the Rab protein family in apicomplexan parasites. Microbes Infect 10: 462-470.

Matrajt M, Donald RG, Singh U, Roos DS 2002. Identification and characterization of differentiation mutants in the protozoan parasite Toxoplasma gondii. Mol Microbiol 44: 735-747.

Mazumdar J, Wilson EH, Masek K, Hunter CA, Striepen B 2006. Apicoplast fatty acid synthesis is essential for organelle biogenesis and parasite survival in Toxoplasma gondii. Proc Natl Acad Sci USA 103: 13192-13197.

Meissner M, Agop-Nersesian C, Sullivan WJ Jr 2007a. Molecular tools for analysis of gene function in parasitic microorganisms. Appl Microbiol Biotechnol 75: 963-975.

Meissner M, Breinich MS, Gilson PR, Crabb BS 2007b. Molecular genetic tools in Toxoplasma and Plasmodium: achievements and future needs. Curr Opin Microbiol 10: 349-356.

Meissner M, Schluter D, Soldati D 2002. Role of Toxoplasma gondii myosin A in powering parasite gliding and host cell invasion. Science 298: 837-840.

Mital J, Meissner M, Soldati D, Ward GE 2005. Conditional expression of Toxoplasma gondii apical membrane antigen-1 (TgAMA1) demonstrates that TgAMA1 plays a critical role in host cell invasion. Mol Biol Cell 16: 4341-4349.

Plattner F, Yarovinsky F, Romero S, Didry D, Carlier MF, Sher A, Soldati-Favre D 2008. Toxoplasma profilin is essential for host cell invasion and TLR11-dependent induction of an interleukin-12 response. Cell Host Microbe 3: 77-87.

Robibaro B, Stedman TT, Coppens I, Ngo HM, Pypaert M, Bivona T, 
Nam HW, Joiner KA 2002. Toxoplasma gondii Rab5 enhances cholesterol acquisition from host cells. Cell Microbiol 4: 139-152.

Sheiner L, Soldati-Favre D 2008. Protein trafficking inside Toxoplasma gondii. Traffic 9: 636-646.

Soldati D, Boothroyd JC 1993. Transient transfection and expression in the obligate intracellular parasite Toxoplasma gondii. Science 260: $349-352$.

Stedman TT, Sussmann AR, Joiner KA 2003. Toxoplasma gondii Rab6 mediates a retrograde pathway for sorting of constitutively secreted proteins to the Golgi complex. J Biol Chem 278: 5433-5443.

Striepen B, Jordan CN, Reiff S, van Dooren GG 2007. Building the perfect parasite: cell division in Apicomplexa. PLoS Pathog 3: e78.
Striepen B, White MW, Li C, Guerini MN, Malik SB, Logsdon JM Jr, Liu C, Abrahamsen MS 2002. Genetic complementation in apicomplexan parasites. Proc Natl Acad Sci USA 99: 6304-6309.

Ullu E, Tschudi C, Chakraborty T 2004. RNA interference in protozoan parasites. Cell Microbiol 6: 509-519.

Vaishnava S, Striepen B 2006. The cell biology of secondary endosymbiosis-how parasites build, divide and segregate the apicoplast. Mol Microbiol 61: 1380-1387.

Vanchinathan P, Brewer JL, Harb OS, Boothroyd JC, Singh U 2005. Disruption of a locus encoding a nucleolar zinc finger protein decreases tachyzoite-to-bradyzoite differentiation in Toxoplasma gondii. Infect Immun 73: 6680-6688. 